\title{
Dielectric Modulus Relaxation and Hopping Conduction Mechanism of Bisphenol A Corncobs Polycarbonate.
}

\author{
T. A. Hanafy \\ Physics Department, Faculty of Science, Fayoum University, \\ 63514, El Fayoum, Egypt.
}

Dielectric relaxation of bisphenol A corncobs polycarbonate (BPACC) has been studied in the temperature and frequency ranges of $310-400 \mathrm{~K}$ and $100 \mathrm{~Hz}-4 \mathrm{MHz}$, respectively. A clear $\rho, \alpha, \alpha^{\prime}$ and $\beta$-relaxation processes are observed in these studied ranges of temperature and frequency. An analysis of the dielectric constant, $\varepsilon^{\prime}$, and the dielectric loss index, $\varepsilon^{\prime \prime}$, was performed assuming a distribution of relaxation times as confirmed by the behavior of electric modulus spectra. The calculated activation energy, $E_{a}$, are $0.36 \mathrm{eV}$, $0.17 \mathrm{eV}$, and $0.09 \mathrm{eV}$ for $\alpha, \alpha^{\prime}$ and $\beta$-relaxation processes, respectively. The behavior of ac conductivity, $\sigma_{a c}$, of BPACC sample revealed that the relaxation occurs as a result of charge carriers between localized states. The conduction mechanism of BPACC sample is controlled by the correlated barrier hopping model $(\mathrm{CBH})$.

\section{Introduction}

Bisphenol A (BPA) is a valuable raw material used in the production of many polymers and is a fundamental building block of epoxy resins and polycarbonates. The end-products are used in a range of applications; including adhesives, potting compounds, head light covers, and lenses. In the past few years, a rapidly growing demand for bisphenol $\mathrm{A}$ in industrial scale is for use in high-tech production, such as compact discs and digital video discs [1-4]. Bisphenol A corncobs (BPACC) is synthesized through the condensation reaction of BPA and the powder of Egyptian corncobs [5, 6]. Both chemical analysis and IR spectroscopy have shown that BPACC resin has a complicated structure. The resin structure includes furan and lignin hydrolysate units, which are present in a random alternation in the resin chains IR spectroscopy of soda lignin/ bisphenol resin shows the presence of aliphatic methyl, methylene, $\mathrm{O}-\mathrm{CH}_{3}$, and strong $\mathrm{OH}$ groups [7]. 
The dielectric constant $\varepsilon^{\prime}$ and the dielectric loss factor $\varepsilon^{\prime \prime}$ are crucial quantities required in the design of the devices. The temperature and frequency dependence of dielectric properties reveal much information on the chemical and physical state of the polymer [8]. The dielectric behavior of polymer is determined by the charge distribution and also the statistical thermal motion of its polar groups. The study of the dielectric relaxation behavior constitutes a powerful approach for obtaining information about the nature of the molecular motions. In addition, it explains the manner in which these motions are affected by the chemical composition, molecular structure and morphology [9].

In polymer system, there are two main chain motions. The first is the micro-Brownian motion, which occurs at the glass transition temperature $(\mathrm{Tg})$. The second is a more local type motion, which occurs in the glassy state. The detailed mechanism of the second type seems rather uncertain; a local relaxation mode has been proposed to explain it [10]. Where, the time scale shorter than the micro-Brownian motion has been suggested and the main chain can be regarded as a small scale, confined to neighborhood of the local equilibrium conformation. Above $\mathrm{Tg}$, the material become electrically conductive and shows a sharp increase in the dielectric constant as a result of the interfacial polarization. This is due to the trapping of free change carriers at the boundaries, which exist between crystalline and amorphous phases [11].

The electric modulus $\left(\mathrm{M}^{*}\right)$ is defined as the inverse of the complex permittivity $\varepsilon^{*}$ by the following equation $[12,13]$ :

$$
\begin{aligned}
& M^{*}=\frac{1}{\varepsilon^{*}}=\frac{1}{\varepsilon^{\prime}-j \varepsilon^{\prime \prime}}=\frac{\varepsilon^{\prime}}{\varepsilon^{\prime 2}+\varepsilon^{\prime 2}}+j \frac{\varepsilon^{\prime \prime}}{\varepsilon^{\prime 2}+\varepsilon^{\prime 2}} \\
& M^{\prime}=\frac{\varepsilon^{\prime}}{\varepsilon^{\prime 2}+\varepsilon^{\prime 2}} \text { and } M^{\prime \prime}=\frac{\varepsilon^{\prime \prime}}{\varepsilon^{\prime 2}+\varepsilon^{\prime 2}},
\end{aligned}
$$

where $\mathrm{M}^{\prime}$ and $\mathrm{M}^{\prime \prime}$ are the real and the imaginary parts of the electric modulus. Recently electric modulus has been adopted for the investigation of electrical relaxation effects in polymers $[14,15]$. The use of electric modulus offers some advantages in interpreting bulk relaxation process since by its definition variation in the large values of permittivity and conductivity at low frequencies are minimized. Thus, common difficulties like electrode nature and its contact, space charge injection phenomena and absorbed impurity conduction effects, which appear to obscure relaxation in the permittivity presentation, can be resolved or even ignored [16]. 
In this paper we investigate the dielectric relaxation of bisphenol $\mathrm{A}$ corncobs resin in the temperature and frequency ranges of $310-400 \mathrm{~K}$ and 100 $\mathrm{Hz}-5 \mathrm{MHz}$, respectively. The electric modulus representation has been used to provide comparative analysis of the ion transport properties. Finally, the ac conductivity of BPACC was examined to give evidence about the type of conduction mechanism in BPACC sample.

\section{Experimental}

BPACC was prepared by adding the powder of corncobs to the bisphenol $\mathrm{A}$ in the weight ratio $1: 1$ (5g of each) together with $0.566 \mathrm{~g}$ mole $\mathrm{HCl} / 50 \mathrm{ml}$ and maintaining at $95-100^{\circ} \mathrm{C}$ for $8 \mathrm{hr}$. The product was cooled and neutralized with 0.1 of ammonium hydroxyl solution. The sample was washed with a diluted acetic acid (30\%) to remove any bisphenol molecules $[6,7]$. The polycondensate was washed with hot water and allowed to dry. The dry resin was ground to a powder. The chemical structure of BPACC is shown in scheme.1 [7]:

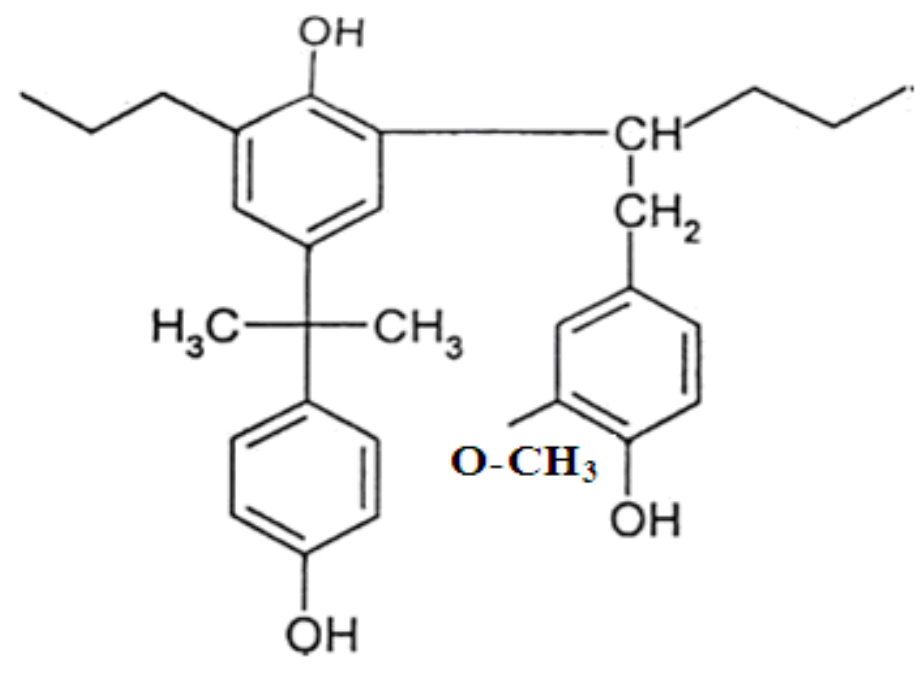

Scheme 1: The chemical structure of BPACC.

A tablet of the investigated sample was obtained by presses $2.0 \mathrm{mg}$ of BPACC under the pressure of $1.96 \times 10^{8} \mathrm{~N} / \mathrm{m}^{2}$. The obtained BPACC sample has a radius and a thickness of $0.45 \mathrm{~cm}$ and $1.3 \mathrm{~mm}$, respectively. Dielectric spectroscopy measurements were accomplished using a Hioki (Ueda, Nagano, Japan) model 3532 High Tester LCR, with the accuracy of order $\pm 0.08 \%$. The dielectric constant, $\varepsilon^{\prime}$, and dielectric loss factor, $\varepsilon^{\prime \prime}$, were recorded at frequency and temperature ranging from $100 \mathrm{~Hz}$ to $5 \mathrm{MHz}$ and $300-400 \mathrm{~K}$, respectively. $\varepsilon^{\prime}$, and $\varepsilon "$ were calculated as follows: 


$$
\begin{aligned}
\varepsilon^{\prime} & =\frac{C d}{\varepsilon_{o} A} \\
\varepsilon^{\prime \prime} & =\varepsilon^{\prime} \tan (\delta)
\end{aligned}
$$

where $\mathrm{C}$ is the capacitance of the sample-filled capacitor, $\mathrm{d}$ is the sample thickness, $\varepsilon_{\mathrm{o}}$ is the vacuum permittivity, and $\mathrm{A}$ is the electrode area. The temperature of the sample was measured with a T-type thermocouple. The measurement accuracy for the temperature was about $\pm 1 \mathrm{~K}$.

\section{Results and discussion:}

Figure 1(a,b) shows temperature dependence of $\varepsilon^{\prime}$ and $\varepsilon^{\prime \prime}$ for bisphenol A corncobs polycarbonate (BPACC) at some selected frequencies. It is clear that, the values of $\varepsilon^{\prime}$ of the investigated sample decrease with the increase of the field frequency. This can be discussed as follows: at low frequency the dipole of the sample can follow the direction of the electric field but at very high field frequency it can no longer follow the applied field. Then, the decrease of $\varepsilon^{\prime}$ with the increase of the field frequency can be assigned to the reduction of the number of the dipoles which contribute to the polarization process. Also, the behavior of $\varepsilon^{\prime}$ and $\varepsilon^{\prime \prime}$ undergoes two relaxation processes namely $\alpha$ and $\alpha^{\prime}$ relaxation one at 340 and $363 \mathrm{~K}$, respectively. $\alpha$-peak centered at $337 \mathrm{~K}$ for $\varepsilon^{\prime}(\mathrm{T})$ and at $340 \mathrm{~K}$ for $\varepsilon^{\prime \prime}(\mathrm{T})$. This process corresponds to the segmental relaxation which associated with the glass-rubber transition temperature, $\mathrm{Tg}$. At this temperature microBrownian motion of long chain segments in the amorphous regions of BPACC takes place.

The $\alpha^{\prime}$-relaxation process which located at $363 \mathrm{~K}$ for $\varepsilon "(T)$ is related to the segmental motion of the polar groups in crystalline phases of BPACC structure. This process is probably due to the dipole relaxation within the crystalline or in the regions intermediate between the amorphous and the crystalline phases of the backbone structure of BPACC sample. Also, above 370K there is increase for $\varepsilon^{\prime}$ and $\varepsilon "$ at low frequency. This effect can be attributed to the motion of space charges that are accumulated in the polymer close to the electrode during formation. This indicates that the behavior of $\varepsilon^{\prime \prime}$ undergoes $\rho$-relaxation process. On the other hand the increase of $\varepsilon^{\prime}$ at low frequency and at higher temperature can be assigned to the motion of the free charge carriers within the sample. It moves thoroughly the amorphous phase of BPACC structure. These carriers are hindered in their motion by the crystalline phases of the sample and pile up at the interface between the amorphous and the crystalline phases. This causes the 
build-up of a macroscopic charge separation or space charge polarization $[15$, $16]$.

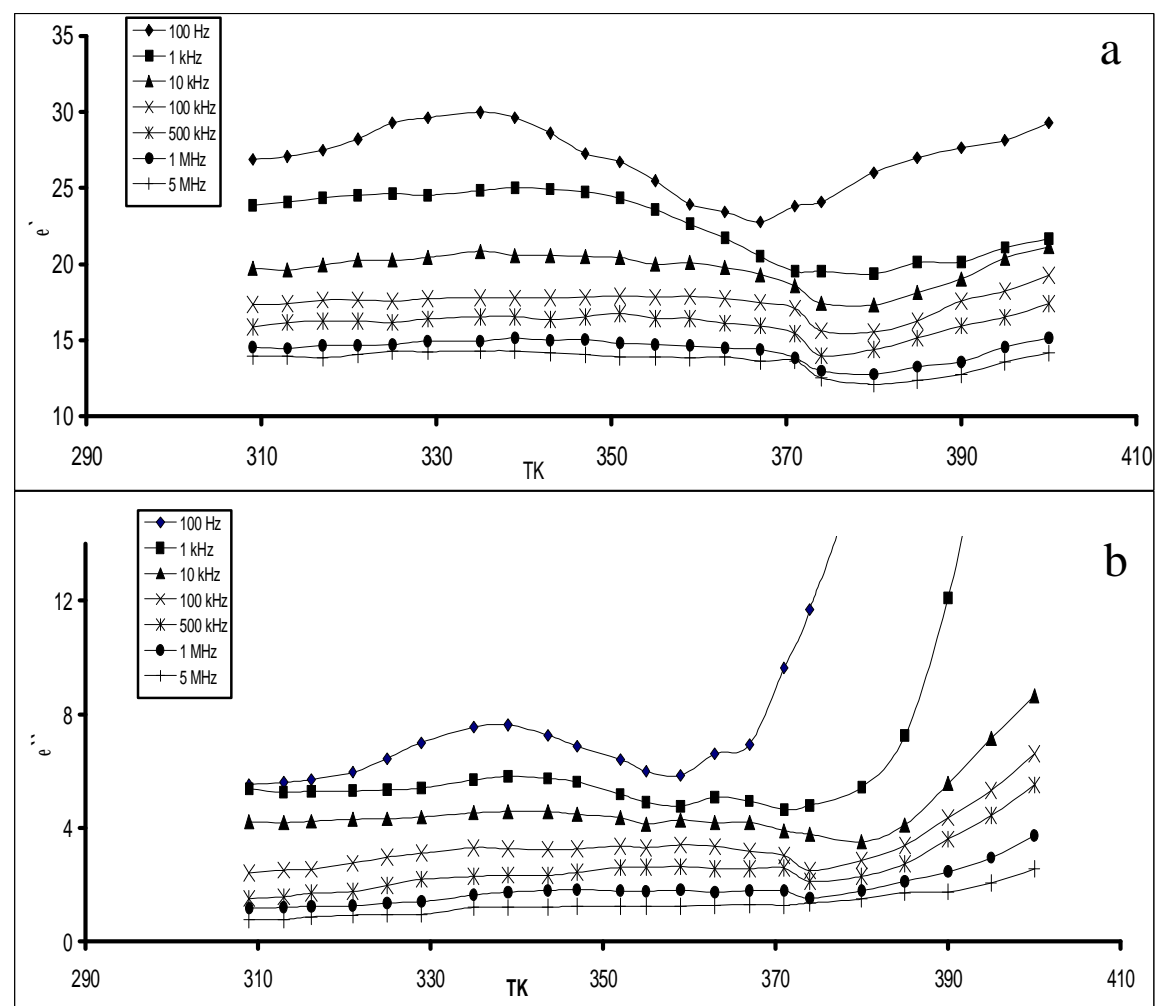

Fig. 1(a,b): Temperature dependence of $\varepsilon^{\prime}$ and $\varepsilon^{\prime \prime}$ for BPACC sample at some constant frequencies.

Figure 2(a,b) illustrates the frequency dependence of $\varepsilon^{\prime}$ and $\varepsilon^{\prime \prime}$ for BPACC at some fixed temperatures, It is noticed that $\varepsilon^{\prime}(\mathrm{f})$ exhibits two relaxation peaks namely $\alpha$ - and $\beta$-relaxation processes at $200 \mathrm{kHz}$ and $2 \mathrm{MHz}$, respectively. While the behavior of $\varepsilon$ " undergoes three relaxation processes namely $\alpha-, \alpha^{\prime}-$ and $\beta$ peak, at $80 \mathrm{kHz}, 500 \mathrm{kHz}$, and $1.5 \mathrm{MHz}$, respectively. The $\alpha$-relaxation peak is certainly due to the micro-Brownian motion of the main chain of the polymeric material $[17,18]$. This process is assigned to the release of frozen in dipoles by their cooperative motion with adjoining segments of the main chain. The $\alpha^{\prime}$-peak can be ascribed to the rotational polarization of the two phenyl groups of BPACC. So, with the increase of the field frequency the contribution of rotational polarization of phenyl groups which attached directly to main chain of BPACC becomes significant. In addition, the polarization of the two hydroxyl groups plays an important role in this process. 


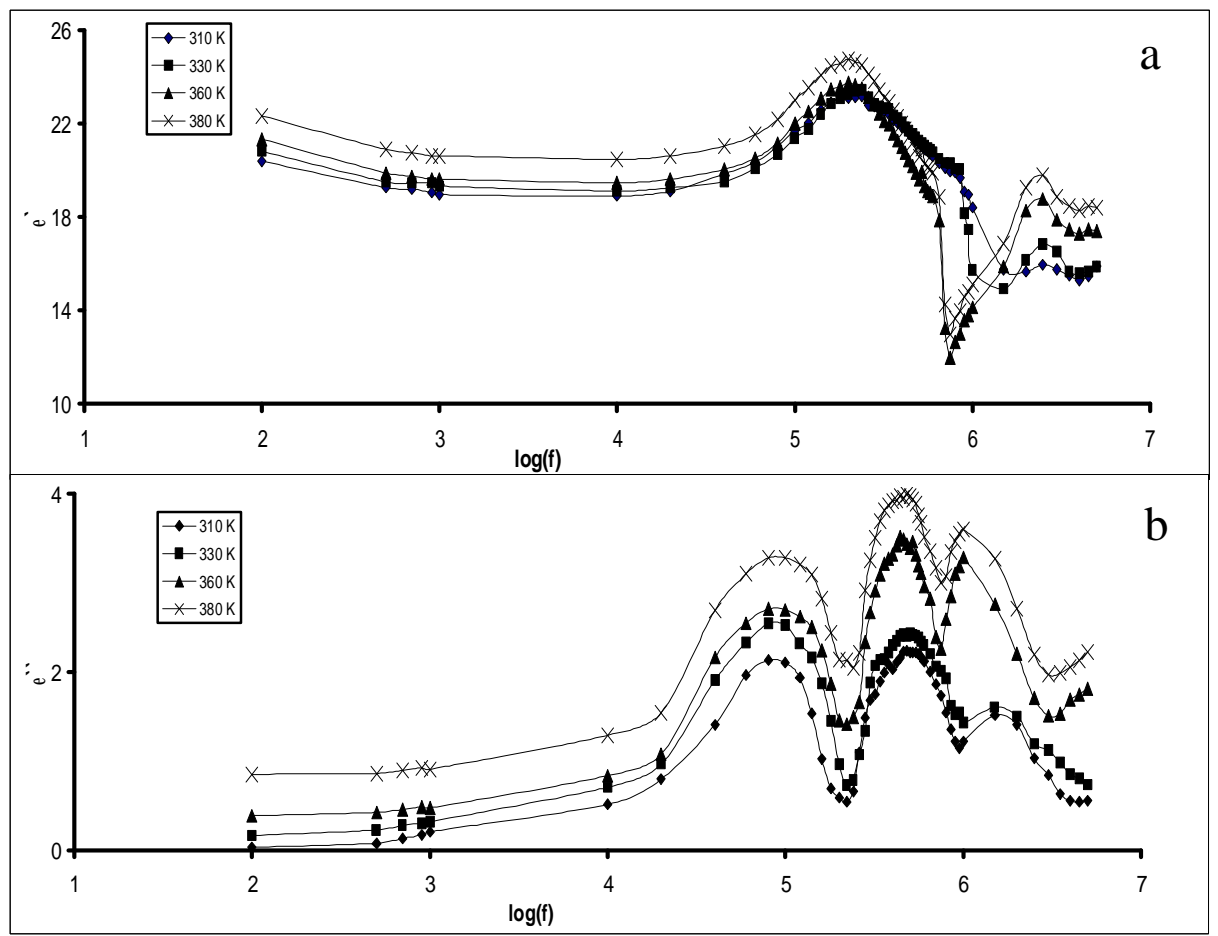

Fig. (a,b): Frequency dependence of $\varepsilon^{\prime}$ and $\varepsilon^{\prime \prime}$ for BPACC sample at some constant temperatures.

$\beta$-relaxation process can be attributed to the local motion of the dipole within the crystalline phases of the investigated sample. The peak height of this process shafts to lower frequency with the increase of the temperature. This means that the length of the order phase reaction is reduced with the increase of the temperature. This agrees with some suggestions in literature [19]. Also, $\beta$-relaxation process is associated with the local motion determined by rotational or vibrational fluctuations of the side groups. So, the polarization of the two aliphatic methyl groups and $\mathrm{O}-\mathrm{CH}_{3}$ has a great influence in the emergence of $\beta$ relaxation.

The temperature dependence of real $\mathrm{M}^{\prime}$ and the imaginary $\mathrm{M}^{\prime \prime}$ parts of complex modulus for BPACC sample at some fixed frequencies is shown in Fig. $3(\mathrm{a}, \mathrm{b})$. It is observed that the values of $\mathrm{M}^{\prime}$ approximately constant with the increase of temperature up to $370 \mathrm{~K}$ at each frequency. This means that the role of the electrode polarization for $\alpha$ - and $\alpha$-relaxation processes is vanish. This indicates that the nature of the dielectric constant of BPACC sample is thermally activated [20, 21]. Above 370K, $\mathbf{M}^{\prime}$ decreases rapidly with the increase of temperature especially at low frequency $(100 \mathrm{~Hz})$. Also, the behavior of M" 
exhibits undergoes three relaxation processes $\alpha, \alpha^{\prime}$ and $\rho$-peaks as mention earlier. The occurring of dielectric relaxations $\alpha, \alpha^{\prime}$ and $\rho$-peaks is the consequence of the efforts carried out by the polar segments of the polymers system, to follow the imposing direction of the applied alternating field. Near Tg the segmental mobility of the polymer is enhanced and the orientation of the large parts of the molecular chains is facilitated.

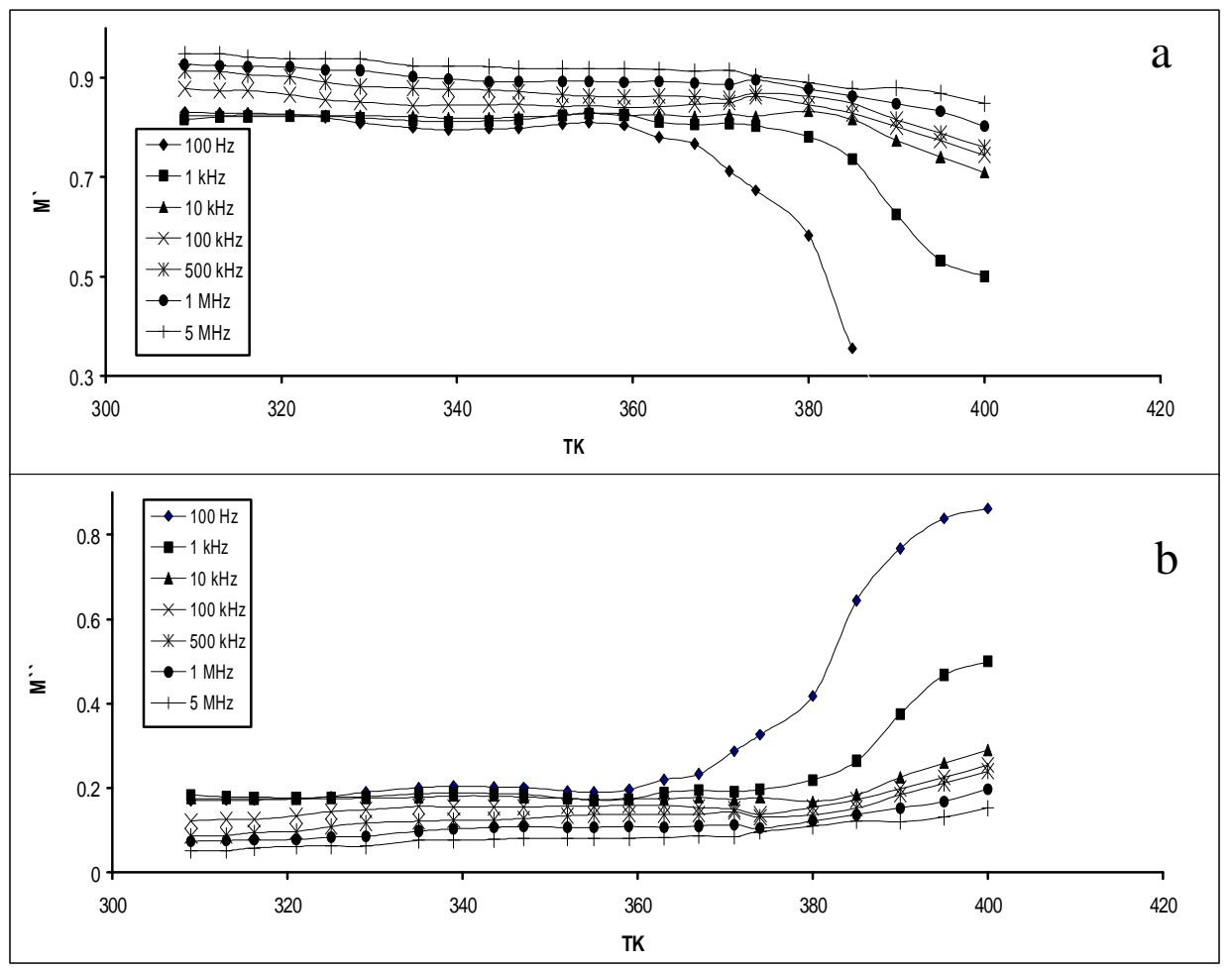

Fig. 3(a,b): Temperature dependence of $\mathrm{M}^{\prime}$ and $\mathrm{M}^{\prime \prime}$ for BPACC sample at some constant frequencies.

At lower temperatures, relaxation occurs due to the orientation of smaller polar groups such as side groups or local segments of the main chain [22]. However, the existence of the $\rho$-relaxation process, at higher temperature and at lower frequencies for BPACC sample, gives rise to the interfacial polarization or Maxwell Wagner Sillars (MWS) effect [23, 24]. The peak height values of M" were found to be lower than obtained for $\varepsilon^{\prime \prime}$ as in Fig. 2(b). This indicates the removal of the electrode polarization. It can be suggested that the $\rho$-relaxation process in BPACC is characterized by a combination of MWS process and dc conductivity. In addition, the free charge carriers which trapped by the surrounding crystallites, accumulate at the interface and move through the amorphous phase under the influence of an applied alternating electric field. 
Figure $4(\mathrm{a}, \mathrm{b})$ depicts the frequency dependence of $\mathrm{M}^{\prime}$ and $\mathrm{M}^{\prime \prime}$ for BPACC sample over the temperature range from $310 \mathrm{~K}$ to $380 \mathrm{~K}$. It is clear that BPACC exhibits three relaxation processes. The first is $\alpha$-peak, located at $80 \mathrm{kHz}$ for M"(f), is due to the reorientation of the polar segments of the polymer main chain during Tg [13]. The second is $\alpha^{\prime}$-peak, located at $500 \mathrm{kHz}$ for M"(f), is due to the reorientation of the polar groups such as phenyl and $\mathrm{OH}$ ones within the crystalline phases of the investigated sample. The third is $\beta$-peak, located at 1.5 $\mathrm{MHz}$ for M"(f), is attributed to the rotational polarization of the two methyl and $\mathrm{O}-\mathrm{CH}_{3}$ groups which contribute to the polarization process at higher frequency.

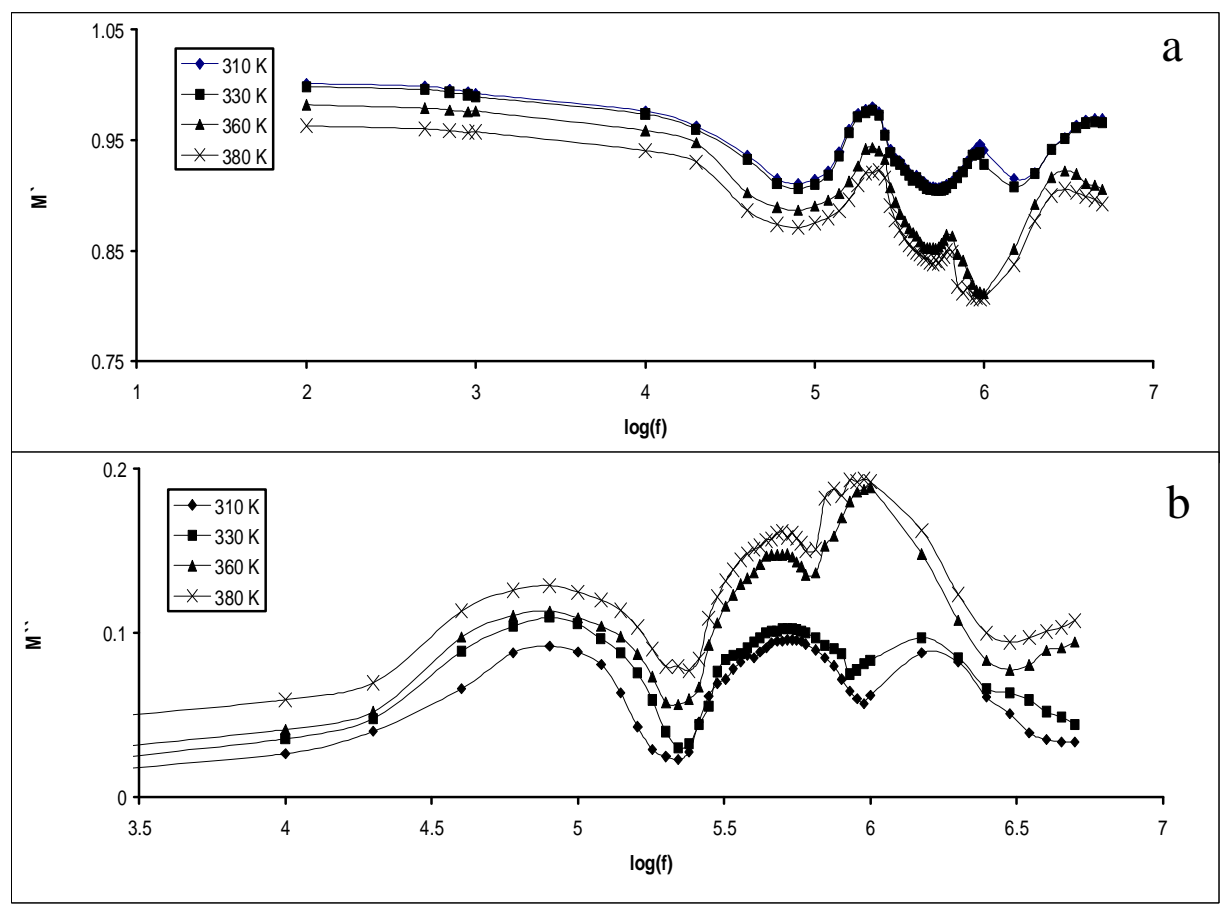

Fig. 4(a,b): Frequency dependence of $\mathrm{M}^{\prime}$ and $\mathrm{M}^{\prime \prime}$ for BPACC sample at some constant temperatures.

The loss curves at varies temperatures for $\alpha, \alpha^{\prime}$ and $\beta$-relaxation processes can be reduced to a master curve by using the peak position $\left(f_{\max }\right)$ and the peak height values $\left(\varepsilon^{\prime \prime}{ }_{\max }\right)$ and $\left(\mathrm{M}^{\prime \prime}{ }_{\max }\right)$. So, the variation of normalized parameters $\left(\varepsilon^{\prime \prime} / \varepsilon^{\prime \prime}{ }_{\max }\right)$ and $\left(\mathrm{M}^{\prime \prime} / \mathrm{M}_{\text {max }}\right)$ as a function of $\log \left(\mathrm{f} / \mathrm{f}_{\max }\right)$ for BPACC sample is shown in Fig. 5. The shape of these curves shows an asymmetric behavior and it broader than that of Debye relaxation. Consequently, a nonsymmetrical distribution of relaxation time exists. The overlap peak position of $\left(\varepsilon^{\prime \prime} / \varepsilon^{\prime \prime}{ }_{\max }\right)$ and $\left(\mathrm{M}^{\prime \prime} / \mathrm{M}_{\text {max }}\right)$ curves is evidence of delocalized or long-range of $\alpha$-relaxation process, Fig. 5 (a). However, for the present system the $\left(\varepsilon^{\prime \prime} / \varepsilon^{\prime \prime}{ }_{\max }\right)$ and 
$\left(\mathrm{M}^{\prime \prime} / \mathrm{M}^{\prime \prime}{ }_{\max }\right)$ peak don't overlap for $\alpha^{\prime}$ and $\beta$-relaxation but are a very close. This suggested that the components from both long range and localized relaxation are present are present [25].
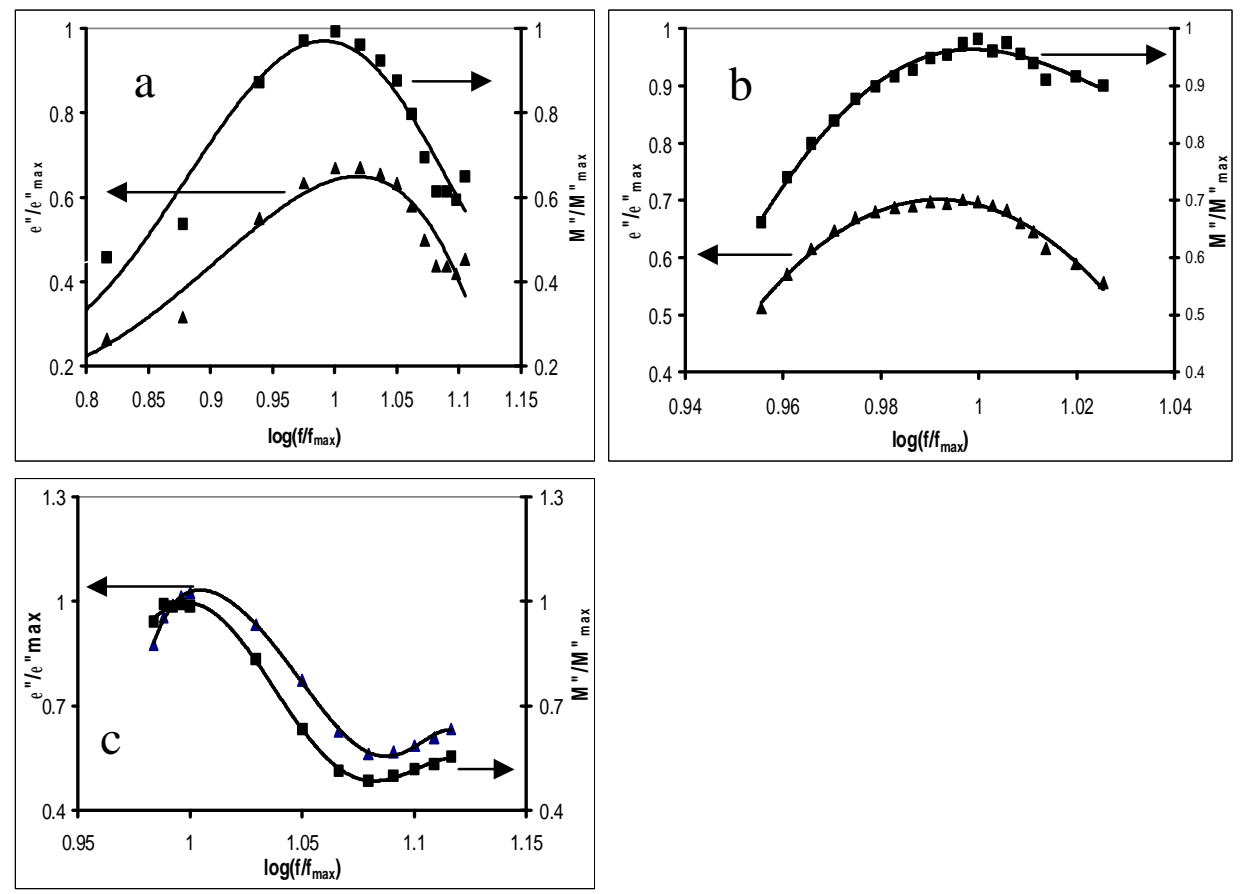

Fig. 5(a-c): (a) The dependence of $\left(\varepsilon^{\prime \prime} / \varepsilon^{\prime \prime} \max \right)$ and $\left(\mathrm{M}^{\prime \prime} / \mathrm{M}^{\prime \prime}{ }_{\max }\right)$ on $\left(\mathrm{f} / \mathrm{f}_{\max }\right)$ for $\alpha$-relaxation process.

(b) The dependence of $\left(\varepsilon " / \varepsilon^{\prime \prime}{ }_{\max }\right)$ and $\left(\mathrm{M}^{\prime \prime} / \mathrm{M}^{\prime \prime}{ }_{\max }\right)$ on $\left(\mathrm{f} / \mathrm{f}_{\max }\right)$ for $\alpha^{\prime}$-relaxation process.

(c) The dependence of $\left(\varepsilon " / \varepsilon^{\prime \prime}{ }_{\max }\right)$ and $\left(\mathrm{M}^{\prime \prime} / \mathrm{M}^{\prime}{ }_{\max }\right)$ on $\left(\mathrm{f} / \mathrm{f}_{\max }\right)$ for $\beta$-relaxation process.

The Cole-Cole plots are good technique in order to investigate the relaxation mechanism in polymers [14, 26, 27]. The Cole-Cole plots of $\varepsilon^{\prime}$ versus $\varepsilon^{\prime \prime}$ of $\alpha, \alpha^{\prime}$ and $\beta$-relaxation for BPACC sample at different temperatures are shown in Fig. 6 (a-c). This semi-circle of Cole-Cole diagram has been used to determine the distribution parameter $(\gamma)$, the effective relaxation time, $\tau_{0}$, and the molecular relaxation time, $\tau$. By knowing of $\gamma$ and $\tau_{0}, \tau$ can be determined by using the relation [14)]:

$$
\frac{U}{V}=\left(\omega \tau_{o}\right)^{1-\gamma}
$$



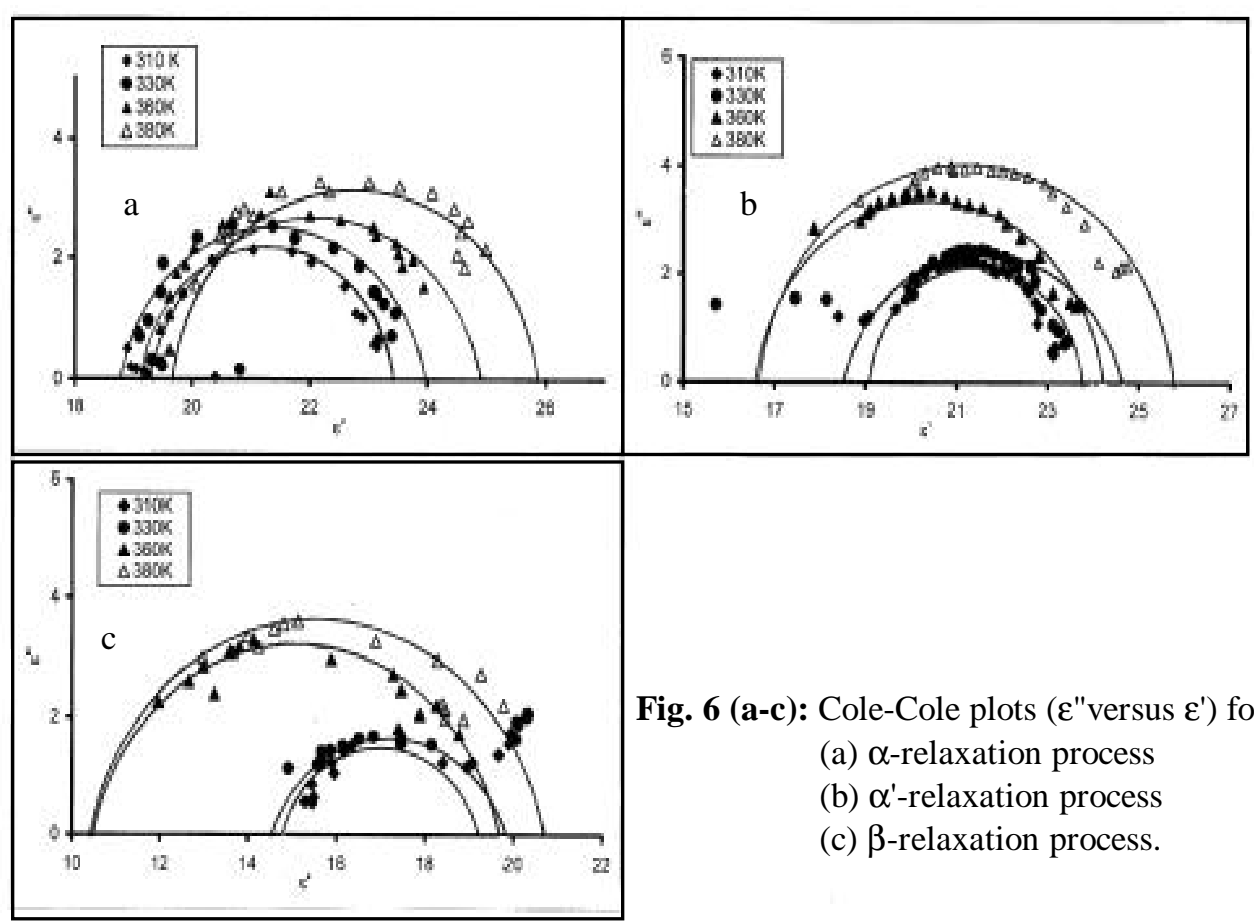

Fig. 6 (a-c): Cole-Cole plots ( $\varepsilon$ "versus $\left.\varepsilon^{\prime}\right)$ for

(a) $\alpha$-relaxation process

(b) $\alpha$-relaxation process

(c) $\beta$-relaxation process.

where $\mathrm{U}$ is the distance from a particular data point in the Cole-Cole plot from the optical dielectric constant $\varepsilon_{\infty}$ and $\mathrm{V}$ is the distance of the same data point from the static dielectric constant $\varepsilon_{\mathrm{s}}$ and $\omega$ is the angular frequency. The parameter $\gamma$ equal zero when the compound has only one relaxation time, whereas for a series of relaxation times, the value of $\gamma$ varies from 0 to 1 . The extent of the distribution of the relaxation time increases with increasing $\gamma$ parameter. The relaxation time, $\tau$, could be calculated by [28]:

$$
\tau=\frac{2 \varepsilon_{o}+\varepsilon_{\infty}}{3 \varepsilon_{o}} \tau_{o}
$$

The values of $\gamma$, and $\tau_{\mathrm{o}}$ Eq 5 are calculated and listed in Table (1). The temperature dependence of $\tau$ for $\alpha, \alpha^{\prime}$ and $\rho$-peaks can be expressed by a thermally activated process in the form of an Arrhenius relation [14, 28]:

$$
\tau=\tau_{o} \exp \left(\frac{E_{a}}{k T}\right)
$$

where $E_{a}$ is the activation energy, $\mathrm{k}$ is the Boltzmann's constant, and $\mathrm{T}$ is the absolute temperature. Fig. (7) represents the reciprocal temperature dependence of $\tau$. The calculated values of $\mathrm{E}_{\mathrm{a}}$ were found to be $0.36 \mathrm{eV}, 0.17 \mathrm{eV}$ and $0.09 \mathrm{eV}$ 
for $\alpha, \alpha^{\prime}$ and $\beta$-relaxation processes, respectively. The values of $E_{a}$ are in good agreement with the reported data for the activation energy $\alpha, \alpha^{\prime}$ and $\beta$-relaxation processes [29-31].

Table 1: The values of $\gamma$-parameter and $\tau_{\mathrm{o}}$ for BACC sample.

\begin{tabular}{|c|c|c|c|c|c|c|}
\hline T K & $\gamma_{\alpha \text {-process }}$ & $\gamma_{\alpha^{\prime} \text {-process }}$ & $\gamma_{\beta \text {-process }}$ & $\begin{array}{l}\tau_{\mathrm{o} \alpha \text {-process }} \\
\mathrm{X} 10^{-6} \mathrm{sec}\end{array}$ & $\begin{array}{l}\tau_{\mathrm{o}} \alpha^{\prime} \text {-process } \\
\mathrm{X} 10^{-7} \mathrm{sec}\end{array}$ & $\begin{array}{c}\tau_{\mathrm{o} \beta \text {-process }} \\
\mathrm{X} 10^{-8} \mathrm{sec}\end{array}$ \\
\hline 310 & 0.13 & 0.07 & 0.22 & 9.00 & 3.18 & 5.40 \\
\hline 330 & 0.10 & 0.07 & 0.244 & 3.80 & 3.00 & 0.50 \\
\hline 360 & 0.05 & 0.05 & 0.13 & 3.62 & 3.80 & 0.82 \\
\hline 380 & 0.05 & 0.30 & 0.44 & 0.67 & 2.40 & 0.47 \\
\hline
\end{tabular}

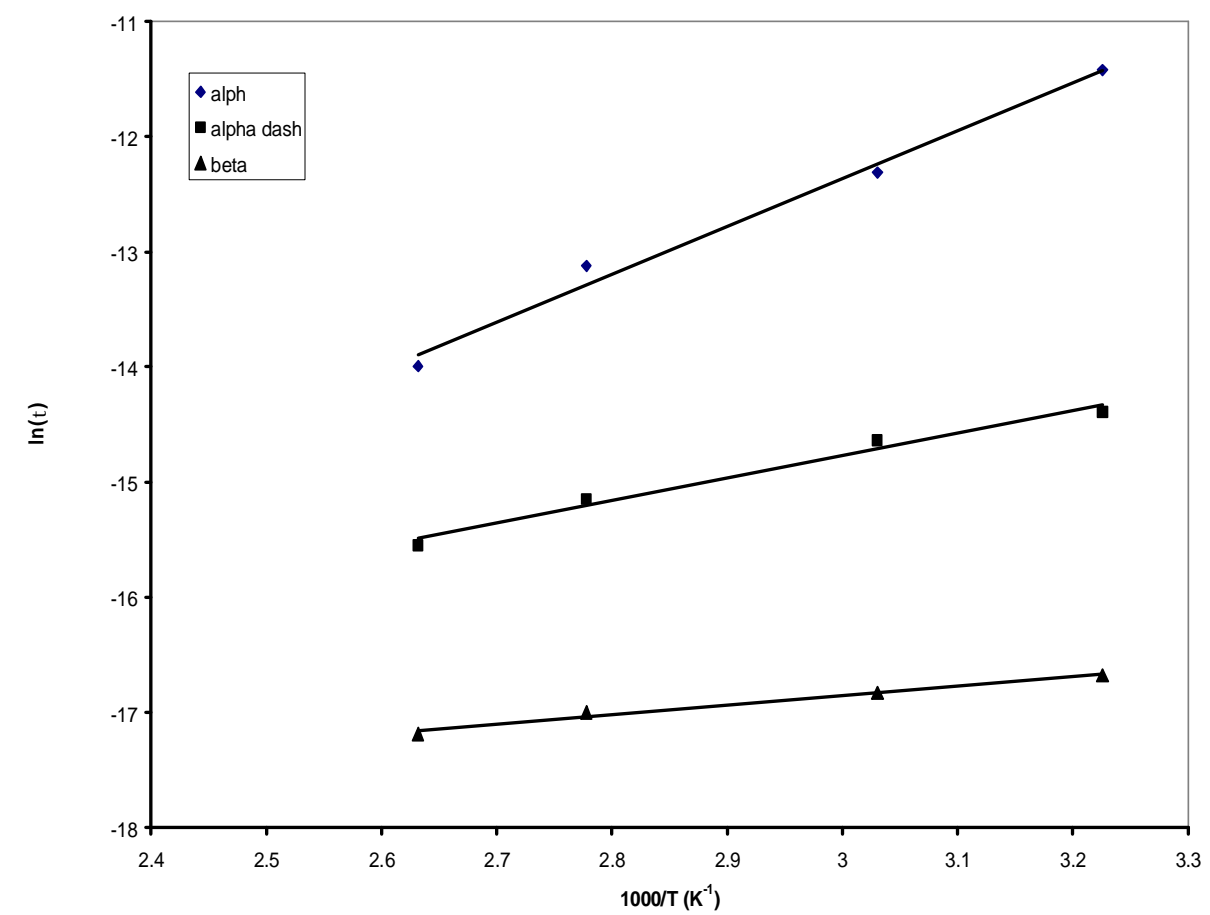

Fig. (7): The reciprocal temperature dependence of relaxation time $(\tau)$.

Figure (8) shows the temperature dependence of the dielectric strength, $\Delta \varepsilon$, of the investigated sample. It is clear that the values of $\Delta \varepsilon$ for $\alpha, \alpha^{\prime}$ and $\beta$-relaxation processes increase with the increase of temperature. This can be discussed as follows: the increase of the temperature leads to increase of the molecular mobility of BPACC sample. This will assist the dipoles or the polar groups to orient toward the direction of the applied electric field. This will 
increase the peak intensity for $\alpha, \alpha^{\prime}$ and $\beta$-relaxation processes. For the dipolar nature of the relaxation phenomenon, a decrease of $\Delta \varepsilon$ is expected according to Curie's law $(\Delta \varepsilon \alpha 1 / \mathrm{T})$ [32]. Consequently, this is contrary to the behavior observed in Fig. 8. So, it can suggest that the magnitude of $\Delta \varepsilon$ depends on not only the dipole concentration and its dipole moment but also is governed by the inter-chain interaction between adjacent chains. These interactions become weaker with the increase of the ambient temperature. So that the decrease of the effective dipole moment due to Curie's law is more than balanced by the diminishing inter-chain constraints. In addition the lateral movements of the two methyl groups effectively hindered the reorientation of the polar groups, such as phenyl, $\mathrm{O}-\mathrm{CH}_{3}$ and $\mathrm{OH}$ ones of BPACC structure. Consequently, a moderate net increase of $\Delta \varepsilon$ with the increase of temperature is observed.

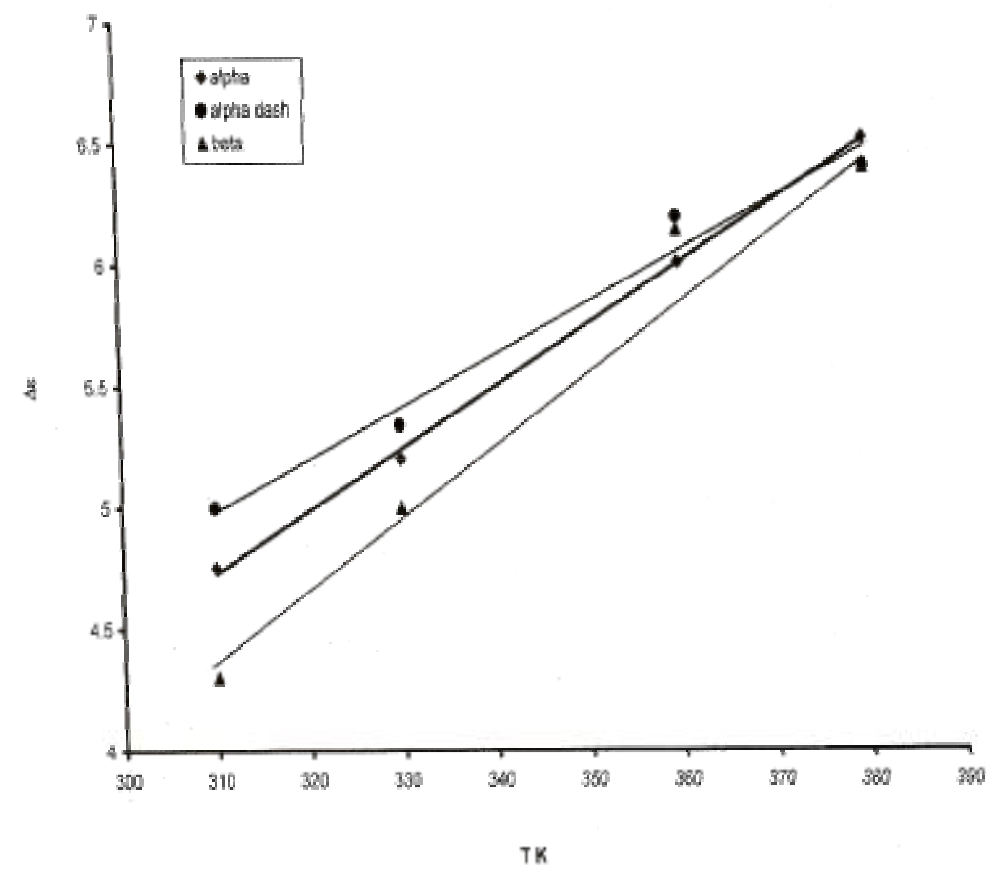

Fig. (8): The temperature dependence of $\Delta \varepsilon$ for BACC sample.

The frequency dependence of ac conductivity $\sigma_{\mathrm{ac}}$ at low temperature side of $\alpha$-relaxation process at some fixed temperatures for BPACC sample is shown in Fig. 9(a). The behavior of $\sigma_{\mathrm{ac}}$ as a function of the field frequency at low temperature is described by [23, 33, 34]:

$$
\sigma_{a c}=A \omega^{s}
$$


where $\mathrm{A}$ is constant, $\omega$ is the angular frequency and $\mathrm{s}$ is a fractional exponent between 0 and 1 . In general the study of the temperature dependence of $\mathrm{s}$ is expected to be helpful in elucidating the microscopic dielectric mechanism responsible for ac conductivity. Several models based on the relaxation caused by the hopping or tunneling of electrons between equilibrium sites have been developed to explain the frequency and temperature dependence of $\sigma_{\mathrm{ac}}$. However, these models are applicable only within a limited temperature range. The values of $s$ in the present work are obtained from the slopes of Fig. 9(a). Fig. 9 (b) represents the dependence of $\mathrm{s}$ on the temperature. It is clear that the values of $\mathrm{s}$ decreased as the temperature is increased. So, the conduction mechanism of the ac conductivity can be explained in terms of the classical barrier hopping model $(\mathrm{CBH})[33,34]$. For this model, it was supposed that the charge transport between localized states is mainly due to hopping over the potential barrier separating the states. For a single electron transfer the following equation could be achieved [23, 34]:

$$
s=1-\frac{6 k T}{W_{H}}
$$

where $\mathrm{W}_{\mathrm{H}}$ is the effective energy of the barrier for a single electron hopping. The calculated values of $\mathrm{W}_{\mathrm{H}}$ are listed in Table (2). The values of $\mathrm{W}_{\mathrm{H}}$ are nearly equal to $E_{a}$ of $\alpha$-relaxation process, which calculated by using Eq 7. This may be attributed to the existence of the micro-inhomogeneity of BPACC sample [23]. Furthermore, the temperature dependence of $\mathrm{s}$ revealed that the conduction mechanism of $\sigma_{\mathrm{ac}}$ for BPACC sample controlled mainly by the correlated barrier hopping model $\mathrm{CBH}$.

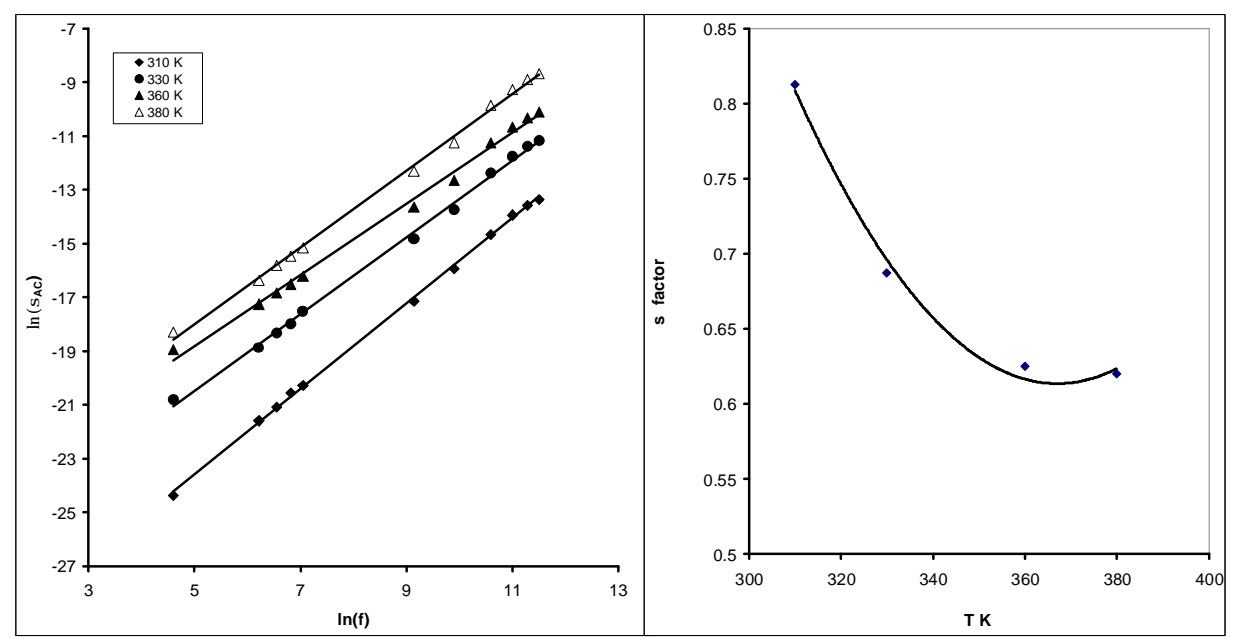

Fig. (9): (a) The frequency dependence of $\sigma_{\mathrm{ac}}$ for $\alpha$-relaxation process.

(b) The temperature of $s$ exponent for $\alpha$-relaxation process. 
Table 2: The values of $\mathrm{W}_{\mathrm{H}}$ for $\alpha$-relaxation process of BPACC sample.

\begin{tabular}{|l|l|}
\hline \multicolumn{1}{|c|}{ T K } & \multicolumn{1}{|c|}{$\mathrm{W}_{\mathrm{H}} \mathrm{eV}$} \\
\hline 310 & 0.84 \\
\hline 330 & 0.54 \\
\hline 360 & 0.49 \\
\hline 380 & 0.51 \\
\hline
\end{tabular}

\section{Conclusion}

The dielectric investigation of BPACC revealed that the existence of four relaxation processes namely $\rho, \alpha, \alpha^{\prime}$ and $\beta$. The first is due to the space charge transition which due to the chain trapping at the interfaces or MWS polarization. The second is due to micro-Brownian motion of the segmental motion of the main chain of the BPACC. The third is assign to the segmental motion of the phenyl and hydroxyl groups. The fourth is attributed to the local motion of the dipolar groups such as methyl and $\mathrm{O}-\mathrm{CH}_{3}$. Both dielectric constant and electric modulus relaxations show that BPACC sample has a distribution times. An analysis of $\sigma_{\mathrm{ac}}$ gives evidence about the conduction mechanism for BPACC. The hopping of charge carriers between localized states is dominant conduction mechanism. Finally, the behavior of $\sigma_{\mathrm{ac}}$ conductivity is controlled by $\mathrm{CBH}$ model.

\section{References}

1. C. C. Chen, S. Cheng, L.Y. Jang, Microp. Mesop. Materials, 109, 258 (2008).

2. A. Alamdari, S. Shariat Nasab, A. Jahanmiri, Chem. Eng. Res. Des 88, 1615 (2010).

3. Y. Xiao, R. Liu, L. Xing, Y. Xu, L. Shang, W. Hao, Toxicology in vitro 25, 153 (2011).

4. C. R. Ispas, T. M. T. Ravalli, A. Steere, S. Andreescu, Water. Res. 44, 1961(2010).

5. S. M. Sayyah, A. I. Sabry, I. A. Sabbah, N. E. Metwally, Acta. Polymerica 42, 670 (1991).

6. I. A. Sabbah, F. A. Khalifa, N. E. Metwally, S. M. sayyah, Egypt. J. Chem., 35, 651(1992).

7. M. A. Ahmed, T. A. Hanafy, J. Appl. Poly. Sci., 109, 182 (2008).

8. A. El-Shekeil, H. Al-Mogdama, A. Al-Karbooly, M. M. Khalid, Polymer, 40, 2879 (1999).

9. F. H. Abd El-Kader, W. H. Osman, K. H. Mahmoud, MAF. Basha, Phsica B: 403, 3473 (2008). 
10. S. Mahros, Poly. Inter. 40, 261 (1996).

11. P. A. M. Steeman, F. H. J. Mourer, Polymer, 33, 4236 (1992).

12. N. G. Mc Crum, B. E. Read, G. Williams "An elastic and dielectric effects in polymeric solids" London: Wiley 1967).

13. S. Mahros, T. A. Hanafy, J. Appl. Poly. Sci., 113, 316 (2009).

14. T. A. Hanafy, K. Elbanna, S. El-Sayed, A. Hassen, J. Appl. Poly. Sci., 121, 3306 (2011).

15. P. A. M. Steeman, F. H. J. Maurer, Polymer, 33, 20 (1992).

16. A. Tripathi, A. K. Tripathi, P. K. C. Pillai, J. Mater. Sci., 25, 1947 (1990).

17. G. A. Kumar, Mater. Let., 55, 364 (2002).

18. I. S. Elashmawi, E. M. Abd elrazek, H. M. Ragab, N. A. Hakeem, Phys. B 405, 94 (2010).

19. N. Guarrotxena, M. DE Frutos, J. Retes, Eur. Poly. J 41, 2824 (2005).

20. S. Mahrous, T. A. Hanafy, M. S. Sobhy, Cur. Appl. Phys., 7, 629 (2007).

21. A. K. Jonscher" Dielectric Relaxation in Solids" Chelsea Dielectrics Press, London (1983).

22. G. C. Psarras, E. Manolakaki, G. M. Tsangaris, Compo. A 34, 1187 (2003).

23. T. A. Hanafy, J. Appl. Poly. Sci. 108, 2540 (2008).

24. L. K. H. Van Beek "Dielectric behavior of heterogeneous system" In: J. B. Birks editor. Progress in dielectrics, London: Heywood (1967).

25. Alo. Dutta, T. P. Sinha, Phys. Rev. B 76, 155113 (2007).

26. Y. Y. Pittini, D. Daneshvari, R. Pittini, S. Vaucher, L. Rohr, S. Leparoux, H. Leuenberger, Eur. Poly. J, 44, 1191 (2008).

27. E. El. Shafee, Eur. Poly. J, 38, 413 (2002).

28. M. S. Masoud, N. A. Shaker, A. E. Ali, Spec. Act. A, 65, 127 (2006).

29. M. E. Bassiouni, F. Al-Shamy, N. K. Madi, M. E. Kassem, Mater. Let. 57, 1595 (2003).

30. Ch. V. Subba Redody, Xia. Han, Quan YaoZhu, Li Qiang Mai, Wen Chen, Micelect, Eng. 83, 281 (2006).

31. R. Singh, J. Kumar, P. K. Singh, A. Kaur, R. D. P. Sinha, N. P. Gupta, Polymer, 47, 5919 (2006).

32. S. Mahrous, Inter. J. poly. Mater. 53, 1 (2004).

33. S. R. Elliott, Adv. Phys., 36, 135 (1887).

34. F. H. Abd El Kader, W. H. Osman, K. H. Mahmoud, M. A. F. Basha, Phys. B 403, 3473 (2008). 